\title{
RESEÑA \\ XII Congreso Internacional de Investigación Turística Academia Mexicana de Investigación Turística (Амıт)
}

José F. Domínguez Estrada Universidad del Caribe fdominguez@ucaribe.edu.mx

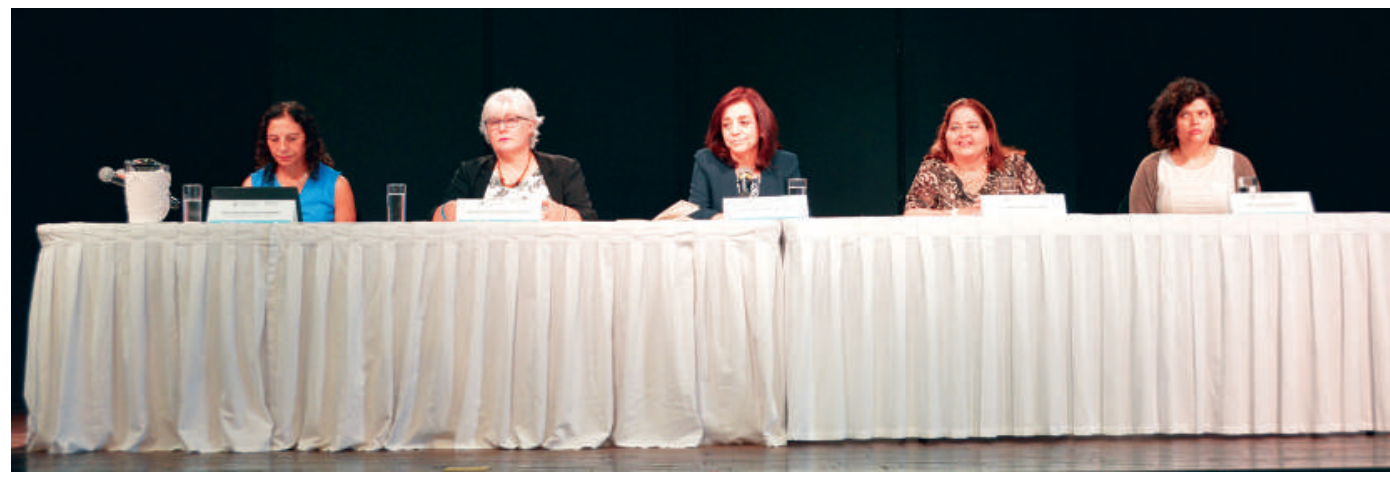

$\int$ on el tema central: "El turismo frente a los objetivos de desarrollo sustentable", la Academia Mexicana de Investigación Turística (AMIT), la Universidad del Caribe (Unicaribe) y la Red de Confluencia Latinoamericana de Investigación Turística (CLAIT) convocaron al XII Congreso Internacional de la AMIT, al XVI Seminario de Turismo y Sustentabilidad de la Unicaribe y al VIII Congreso CLAIT, del 28 al 31 de octubre de 2018 en la ciudad de Cancún, Quintana Roo, México. En la ceremonia de inauguración participaron la Mtra. Teresa Solís Trejo, subsecretaria de Planeación y Política Turística de la Secretaría de Turismo federal, la Mtra. Marisol Vanegas Pérez, secretaria de Turismo del Gobierno del Estado de Quintana Roo, la Dra. Andrea Muñoz Barriga, presidenta del CLAIT, la Dra. Nora L. Bringas Rábago, presidenta de la AmIt, y la anfitriona, la Dra. Ana Pricila Sosa Ferreira, rectora de la Universidad del Caribe. Este congreso fue el marco ideal para intercambiar ideas y reflexionar sobre la contribución real y potencial del turismo al desarrollo sostenible desde una perspectiva crítica.

Durante el congreso, las presentaciones orales se organizaron bajo los siguientes ejes temáticos: 1) Bases epistemológicas y teóricas del turismo; 2) Educación, formación y capacitación en turismo, 3) Comunicación y tecnologías de la información en turismo; 4) Estudios empresariales y administrativos del turismo; 5) Planeación y gestión del turismo; 6) Impactos socio territoriales y ambientales del turismo; 7) Turismo sustentable, de naturaleza o en áreas naturales; 8) Turismo rural, agroturismo o turismo comunitario; 


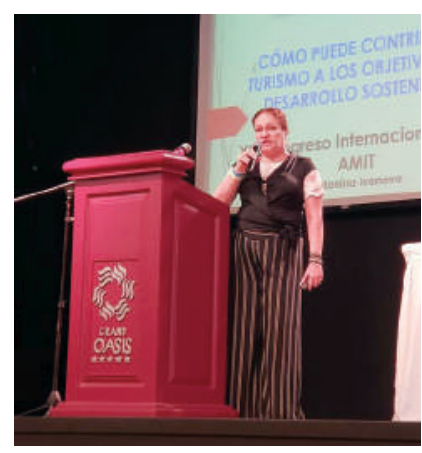

Dra. Antonina Ivanova Boncheva, investigadora de la Universidad Autónoma de Baja California Sur

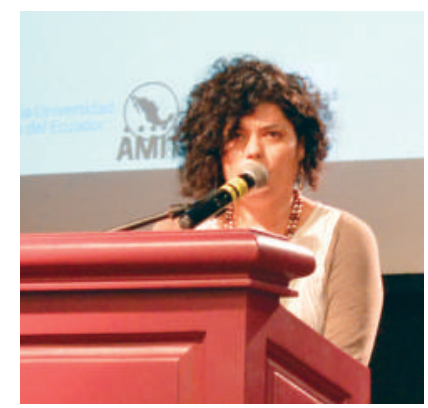

Dra. Andrea Muñoz Barriga. Conferencia magistral: Contradicciones del turismo para la conservación y desarrollo en el paraíso. Galápagos, Ecuador
9) Turismo, cultura y patrimonio; 10) Políticas públicas en turismo, 11) Turismo médico o de salud y turismo deportivo; 12) Metodologías cualitativas y cuantitativas en turismo; 13) Multi, inter y transdisciplinariedad del turismo; y 14) Género y turismo. Adicionalmente se propusieron y presentaron las siguientes mesas especiales: a) Turismo residencial, b) Turismo gastronómico, c) Turismo y parques nacionales; d) Formación superior en turismo y hospitalidad; y e) Turismo alternativo.

Después de la inauguración, la Dra. Antonina Ivanova Boncheva, investigadora de la Universidad Autónoma de Baja California Sur, dio inicio al programa académico con la conferencia magistral: ¿Cómo puede contribuir el turismo a los objetivos del desarrollo sustentable?, e hizo una breve reflexión en torno al tema central del congreso pero con énfasis en la necesidad de visualizar un modelo de desarrollo turístico sustentable más incluyente y al alcance de los sectores más desamparados.

Acto seguido se llevó a cabo el panel: Turismo y Sustentabilidad, coordinado por la Mtra. Marisol Vanegas Pérez, y como participantes el Lic. Francisco Córdova Lira, empresario en Cancún, Quintana Roo; el Mtro. Gonzalo Merediz Alonso, presidente de Amigos de Sian Ka'an; y la C. Zendy Euán, líder comunitaria de la Red de Turismo Comunitario de la Zona Maya. El objetivo del panel fue discutir sobre los logros, obstáculos y pendientes para un turismo sustentable, desde las diversas perspectivas de los actores, de sus programas y proyectos turísticos.

La segunda conferencia magistral estuvo a cargo de la Dra. Andrea Muñoz Barriga, con el tema Contradicciones del turismo para la conservación y desarrollo en el paraíso. Galápagos, Ecuador. La ponente expuso ejemplos claros sobre la preservación y las problemáticas vinculadas con el turismo en esa incomparable área natural protegida de su país.

El segundo panel, fue coordinado por la Dra. Maribel Osorio García, investigadora de la Universidad Autónoma del Estado de México, con el tema Una mirada al turismo desde diferentes disciplinas. El objetivo de este panel fue discutir acera del estudio disciplinario del conocimiento turístico, para reconocer sus alcances y limitaciones e identificar alternativas de fortalecimiento interdisciplinario. Participaron 


\section{J. F. Domínguez Estrada}

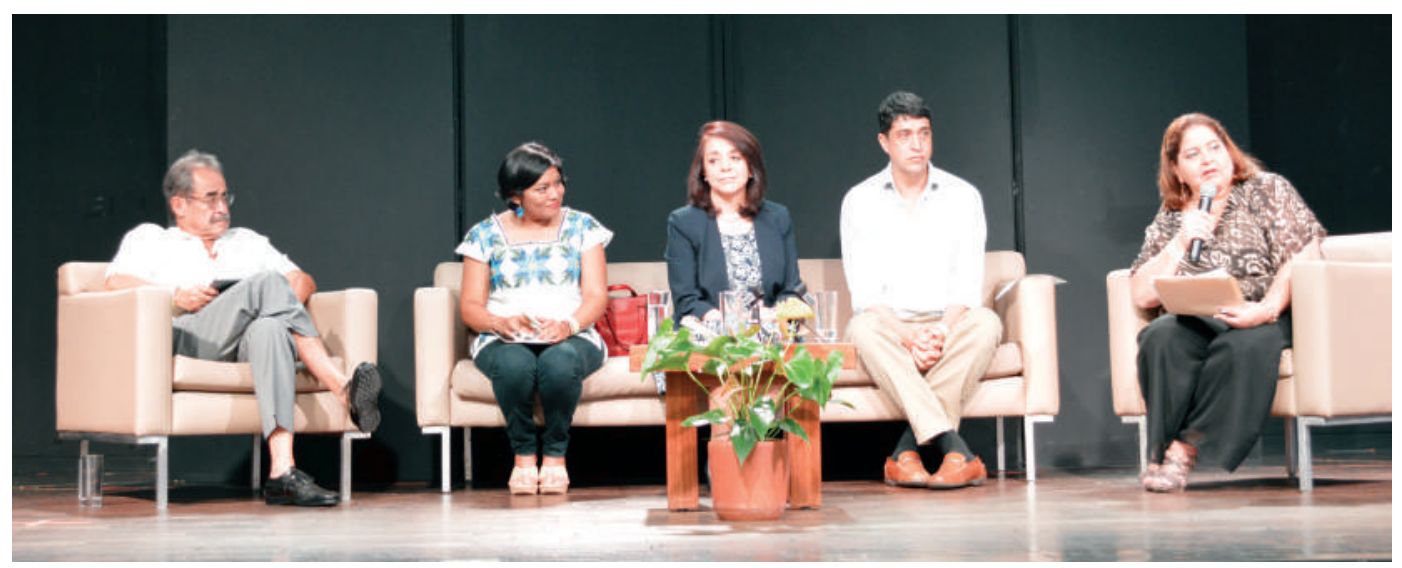

Panel Turismo y sustentabilidad, coordinado por la Mtra. Marisol Vanegas Pérez

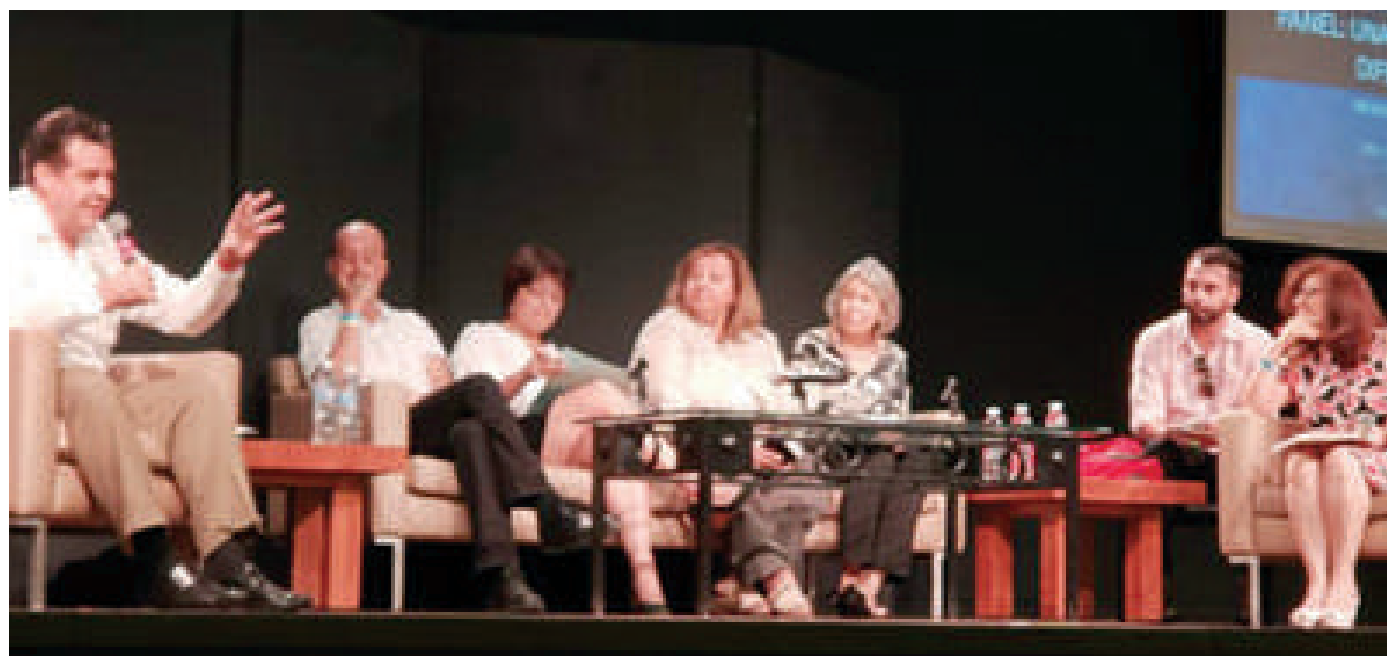

Panel Una mirada al turismo desde diferentes disciplinas, coordinado por la Dra. MariBel Osorio García

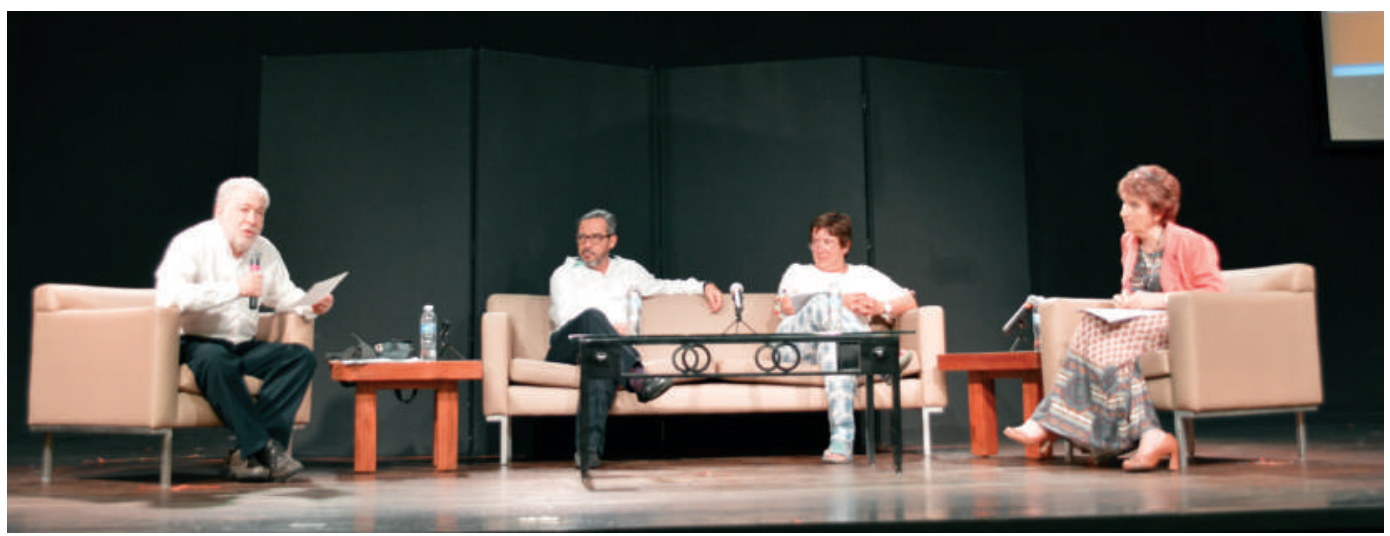

Panel Formación de posgrados en turismo en América Latina: investigación, innovación, internacionalización e interdisciplinariedad, coordinado por el Dr. Pablo Szmulewicz 
Panel Revistas científicas de turismo, coordinado por el Dr. Agustín Santana Talavera, director de la revista PASOS

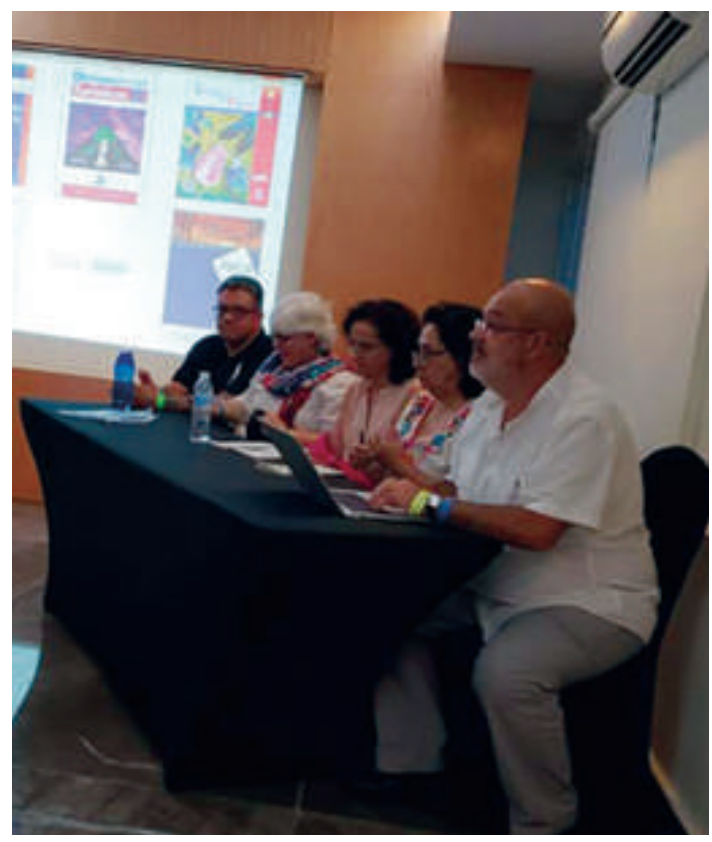

en el evento el Dr. Pedro Moncada Jiménez, secretario Académico de la Universidad del Caribe, por México; el Dr. Alejandro Mantecón Terán, investigador de la Universidad de Alicante, por España; la Mtra. María Alejandra Gazzera, investigadora de la Universidad Nacional del Comahue, por Argentina; la Dra. Mirian Rejowski, investigadora de la Universidad de Sao Paulo, por Brasil; y la Dra. Marisela Janette Pilquiman Vera, investigadora de la Universidad de los Lagos, por Chile.

Para finalizar el primer día de trabajo se celebraron dos paneles simultáneos. El primero con el tema Revistas científicas de turismo, coordinado por el Dr. Agustín Santana Talavera, director de la revista PASOS, y con la participación de la Dra. Rocío del Carmen Serrano Barquín, directora de la revista El Periplo Sustentable; la Dra. Nora L. Bringas Rábago, directora de la revista Dimensiones Turísticas; el Dr. Alejandro Palafox Muñoz, director de la revista Teoría y Praxis; y la Dra. María del Pilar Espeso Molinero, miembro del Comité Editorial de la revista Investigaciones Turísticas. El objetivo de este panel fue analizar la visibilidad de las revistas, las causas del limitado reconocimiento por las agencias evaluadoras, la propuesta de métricas e indicadores para la evaluación de las revistas, la búsqueda de mejoras en los procesos editoriales (disminución en plazos de revisión y publicación, estandarización de un código ético, afrontar el plagio, formas de evitar el sesgo de revisión, etc.) y las formas de financiación del acceso abierto.

El segundo panel, intitulado Formación de posgrados en turismo en América Latina: investigación, innovación, internacionalización e interdisciplinariedad, coordinado por el Dr. Pablo Szmulewicz, director del magíster en Gestión e Innovación del Turismo de la Universidad Austral de Chile, contó con la participación de la Dra. Marcia Capellano do Santos, 


\section{J. F. Domínguez Estrada}

directora del magíster y doctorado en Turismo de la Universidad Caxías do Sul, Brasil; el Dr. Carlos Gauna de León, coordinador de la maestría y doctorado en Ciencias para el Desarrollo, la Sustentabilidad y el Turismo del Centro Universitario de la Costa, Universidad de Guadalajara; y la Mtra. Rossana Campodónico, investigadora de la Universidad de la República en Uruguay y representante del magíster en Turismo de la Universidad de Quilmes, Argentina.

En este panel se analizaron distintas estrategias de fortalecimiento para los programas de posgrado en turismo, especialmente en materia de formación teórico-práctica, de investigación, de capacidades de innovación, de prácticas docentes y de modalidades de titulación innovadoras; así también, se discutieron las vías para la mayor internacionalización de estudiantes y docentes y la formación de redes.

El segundo día tuvo lugar la conferencia magistral El turismo en la teoría social: controversias y desafíos actuales, dictada por el Dr. Alejandro Mantecón Terán, de la Universidad de Alicante, España, a través de la cual reflexionó sobre las perspectivas teóricas aplicadas en el estudio del turismo en los niveles macro o micro y presentó el debate actual sobre estos planteamientos.

Posteriormente se efectuó el foro Los retos de la investigación turística en México, dirigido por la Dra. Nora L. Bringas Rábago y el Dr. Basilio Verduzco Chávez, quienes expusieron los resultados de un reciente estudio realizado por ellos entre los sectores académico, público y privado vinculados al turismo, para identificar las fortalezas y debilidades de la investigación turística en México.

El tercer y último día del Congreso, se llevó a cabo el panel Redes de investigación, dirigido por la Dra. Marta Gemma Nel-Lo Andreu, líder de la Red Internacional de Investigadores en Turismo, Cooperación y Desarrollo (Coodtur); se contó con la participación de la Dra. Alba Font Barnet, secretaria científica de la Red Coodtur, la Dra. Bonnie Lucía Campos Cámara, líder de la Red de Estudios Multidisciplinarios del Turismo de la Península de Yucatán, México (Remtur), y la Dra. Ligia Aurora Sierra Sosa, líder de la Red Universitaria de Antropología Aplicada (RUAA). El objetivo de este panel fue generar un espacio de encuentro y reflexión para analizar la importancia y necesidad de investigar

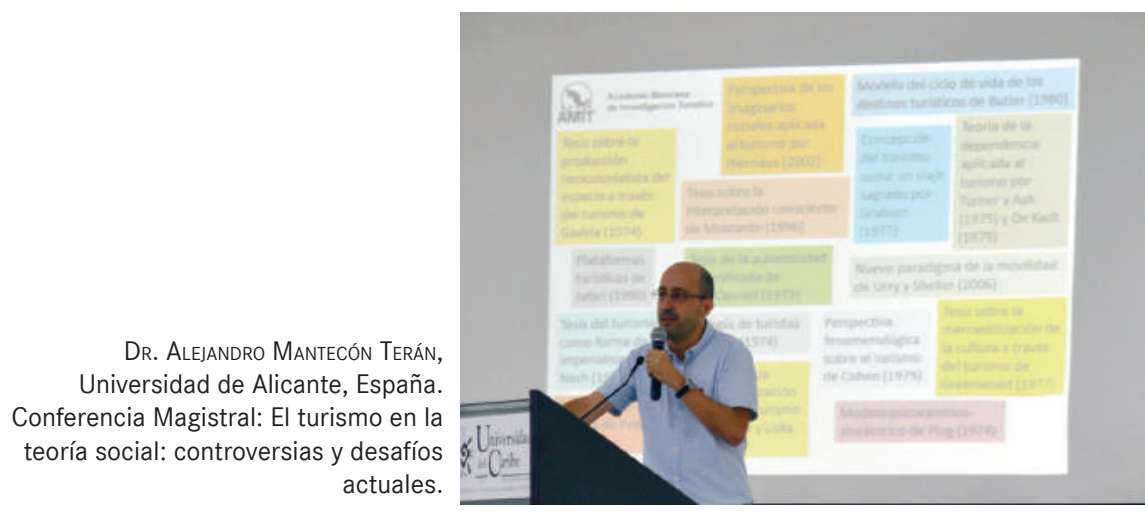




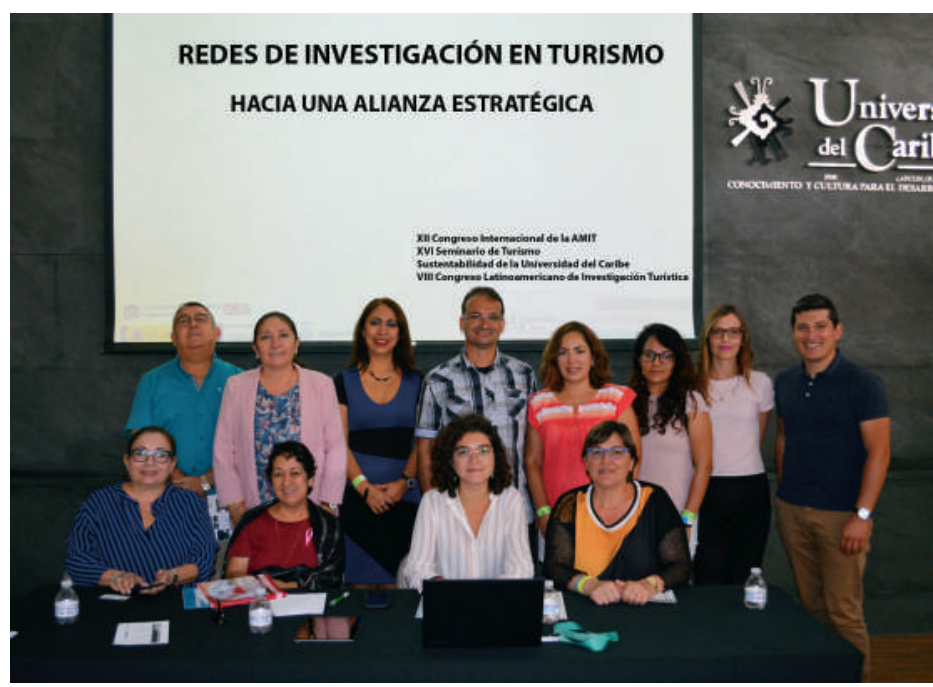

Panel Redes de investigación, dirigido por la Dra. Marta Gemma Nel-Lo Andreu, líder de la Red Internacional de Investigadores en Turismo, Cooperación y Desarrollo

en red, así como aportar criterios para mejorar la calidad, alcance y transferencia de los resultados de la investigación científica en el campo del turismo a nivel internacional.

Para cerrar las conferencia magistrales, la Dra. Raquel Huete Nieves, directora general de Turismo de la Generalitat Valenciana, España, dictó la titulada De la ciudad inteligente al destino turístico inteligente, en la que presentó las acciones llevadas a cabo por la oficina a su cargo para convertir y posicionar a los destinos turísticos localizados en la Comunidad Valenciana -entre ellos el importante centro de Benidorm- en destinos inteligentes destacados en España y en la costa del Mediterráneo; la Dra. Huete Nieves compartió su experiencia, alcances y retos en esta iniciativa.

Durante el Congreso se presentaron también siete libros de destacados investigadores del turismo, la mayoría miembros de la AMIT: Turismo oscuro en México, coordinado por Álvaro López López y Anne Marie Van Broeck; Desarrollo local y turismo en la región de Bahía de Banderas, escrito por María de los Ángeles Huizar Sánchez; Tendencias del turismo en Latinoamérica, de Beatriz Díaz Solano y Tomás J. Cuevas Contreras; Impactos socioculturales del turismo. Dimensiones conceptuales, teóricas y empíricas, de Carlos Monterrubio; Modelo-método holístico Iruma Desu. La innovación a través de las competencias cuantitativas y las competencias cualitativas en la dimensión de lo intangible-cualitativo, de Irma Magaña Carrillo, Karla Y. Covarrubias Cuéllar y Ana B. Uribe Alvarado; Turismo y género. Una mirada desde Iberoamérica, coordinado por Rocío del C. Serrano-Barquín, Gregoria Rodríguez y Daniela Palmas-Castrejón; y Tendencias de investigación en turismo en América Latina. Estudios de caso, coordinado por Andrea Muñoz Barriga, Maribel Osorio y Gabriela Guijarro. 


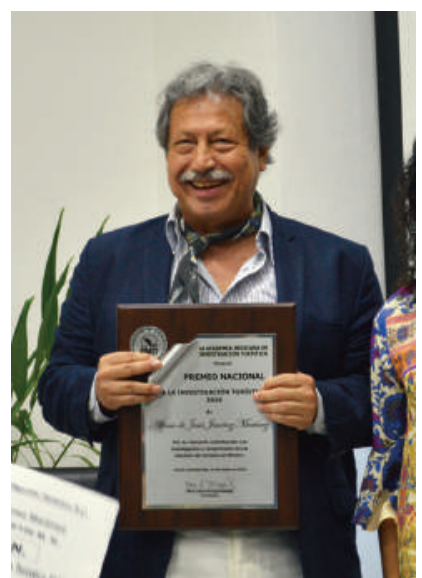

Dr. Alfonso de Jesús Jiménez Martínez, Premio Nacional a la Investigación Turística 2018.

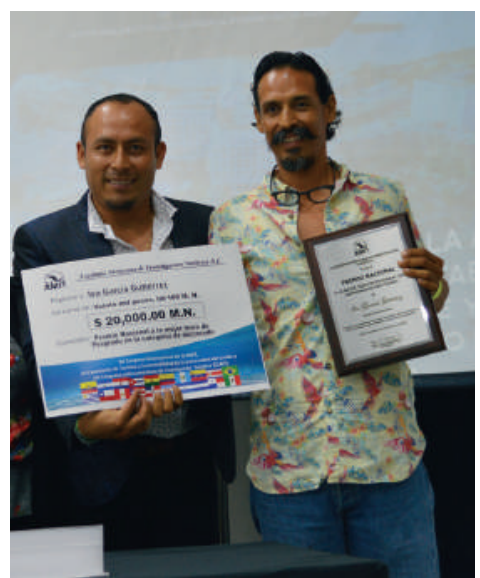

Ivo García Gutiérrez, Premio Nacional a la Mejor Tesis de Doctorado 2017, y su director de tesis Dr. Álvaro López López.

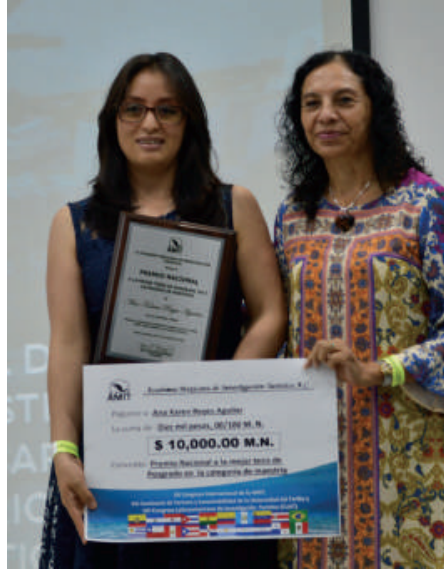

Ana Karen Reyes Aguilar, Premio Nacional a la Mejor Tesis de Maestría 2017, y su directora de tesis Dra. Rocío del Carmen Serrano Barquín.

En el marco de la clausura del Congreso, la AmIT hizo entrega de los siguientes premios. El Premio Nacional a la Investigación Turística 2018 le fue otorgado al Dr. Alfonso de Jesús Jiménez Martínez, quien recibió un diploma de reconocimiento por su trayectoria y la aportación de sus conocimientos a lo largo de cuarenta años para la consolidación de los estudios turísticos y el desarrollo de su investigación, principalmente en el ámbito mexicano, lo que contribuyó a líneas de trabajo importantes académicamente, pero también con una incidencia notable en la transferencia de conocimiento, la mejora de la planificación y políticas turísticas y el esfuerzo en la divulgación. El Dr. Jiménez es profesor-investigador en la Facultad de Turismo de la Universidad Autónoma de Guerrero; adicionalmente recibió un cheque por cuarenta mil pesos.

Igualmente se otorgó el Premio Nacional a la Mejor Tesis de Posgrado 2017, en las categorías de maestría y doctorado. En esta convocatoria se invitó a participar a posgraduados con tesis elaboradas de forma individual por nacionales o extranjeros residentes en el país que concluyeron y defendieron su tesis de grado ante un jurado durante el año 2017, y cuyo tema central se relacionara con el turismo en México. Se recibieron trece participaciones de maestría y cuatro de doctorado de distintas instituciones del país.

En la categoría de doctorado, la tesis premiada fue Impactos socioculturales del turismo asociado al área de protección de flora y fauna Cuatro Ciénagas: Una perspectiva geográfica, de Ivo García Gutiérrez, en el Doctorado en Geografía de la Universidad Nacional Autónoma de México (UNAM); el director de tesis fue el doctor Álvaro López López.

En la categoría de maestría la tesis ganadora fue Mujeres, conservación ambiental y turismo rural en el ejido Adolfo López Mateos, Los Tuxtlas, Veracruz, presentada por Ana Karen Reyes Aguilar, en la Maestría en Ciencias Ambientales de la Universidad Autónoma del Estado de México (UAEM); su directora de tesis fue la Dra. Rocío del Carmen Serrano 
Barquín. Ambos ganadores recibieron un diploma de reconocimiento y un cheque; por diez mil pesos en la categoría de tesis de maestría, y veinte mil pesos en la categoría de la tesis de doctorado.

Como parte del programa recreativo del Congreso se llevó a cabo un brindis de bienvenida, una visita guiada por la ciudad, dos visitas de campo: una a comunidades de Felipe Carrillo Puerto y la otra al Pueblo mágico de Isla Mujeres, y una noche de coctel de despedida en el hotel sede. Estas actividades constituyeron momentos propicios para incentivar la convivencia de los asistentes y los miembros de la AMıT, quienes año tras año se dan cita para intercambiar ideas y resultados de investigación, pero también para encontrar afinidad profesional y personal.

En suma, durante los tres días del evento se presentaron cuatro conferencias magistrales, cuatro paneles, un foro de análisis, 189 ponencias, cuarenta mesas temáticas, diecinueve carteles y siete libros; se congresaron más de 200 especialistas de distintas disciplinas, procedentes de Argentina, Brasil, Colombia, Costa Rica, Chile, España, Ecuador, Uruguay y México, que compartieron sus experiencias y los resultados de sus investigaciones sobre turismo.

Luego de tres días intensos de trabajo, el Congreso llegó a su fin; como parte de este ejercicio intelectual se presentaron las siguientes conclusiones generales. Con respecto al desarrollo teórico de la investigación en turismo y la sustentabilidad, se resaltó que la sustentabilidad fue un hilo conductor del evento y se discutieron aportaciones teóricas y metodológicas en torno al pensamiento de la sustentabilidad como perspectiva de trabajo y como objeto de estudio. Se discutió el concepto de sustentabilidad a la luz del análisis de los retos reales de los actores que toman decisiones en el ámbito público y privado, y se concibió como posible el vincular las conceptualizaciones con las trayectorias profesionales de personas que representan los intereses reales: comunidades, organizaciones cívicas, y empresas, entre otros. Se identificaron retos y avances en la construcción teórica de la relación turismo-desarrollo sustentable, señalando sus cuestionamientos conceptuales, pero a la vez reconociendo las experiencias de la realidad Latino-iberoamericana.

Con relación a los avances metodológicos, se observó que las investigaciones han avanzado en el uso de metodologías mixtas, y que se refleja en mayor medida un trabajo interdisciplinario. Se denotó un mayor nivel de sofisticación metodológica en el estudio de los procesos de la realidad turística, a través de explicaciones causales mejor ordenadas y análisis multivariados cuanti-cuali, reflejando una mayor rigurosidad y consistencia. Sobre la relación investigación-docencia, el evento permitió a estudiantes exponer los avances de sus tesis y recibir retroalimentación de especialistas en el tema, lo que permitió fortalecer su formación de largo plazo. Como parte de este ejercicio de reflexión se discutieron también los alcances y retos de los posgrados en turismo y la necesidad de consolidar su calidad educativa.

Durante el congreso se hizo patente que la comunidad académica congregada está debatiendo sobre diversos temas, entre ellos: la condición de género y la sexualidad, los 


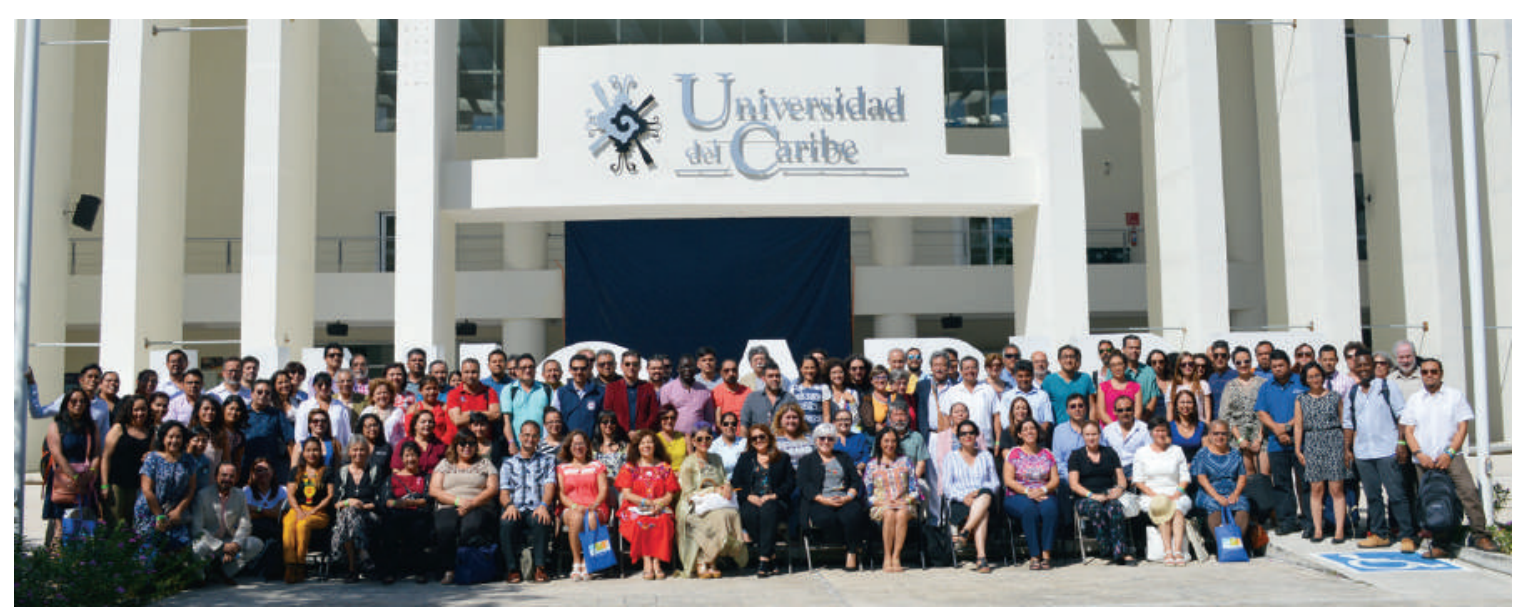

Más de 200 investigadores participaron en el XII Congreso Internacional de Investigación Turística Academia Mexicana de Investigación Turística (Аміт), en el que compartieron sus experiencias y los resultados de sus investigaciones sobre turismo.

procesos de desarrollo local y regional, la organización del territorio y del espacio urbano, los flujos de turistas, el manejo de los sistemas de información y los impactos del uso de las TIC, particularmente reflejado en el interés por los destinos inteligentes y el fenómeno del Airbnb. Sobre la formación de redes y cooperación internacional, se puso de manifiesto el interés de conjuntar esfuerzos y ampliar el diálogo internacional que ayude a compartir discursos y hallazgos de la región Latino-iberoamericana, así como poner en evidencia lo que une a dicha comunidad académica en aspectos como los posgrados, las revistas científicas y las redes de investigación, entre otros.

Finalmente, se dejaron plasmadas distintas recomendaciones sobre la actuación de la comunidad científica del turismo; entre otras, hacer extensiva y permanente la incorporación de tesis en las mesas de trabajo para enriquecer las investigaciones y la formación de recursos humanos; incrementar los diálogos y debates con otras disciplinas y campos, pero también con otros actores y tomadores de decisiones; ampliar nuestras miradas a distintas y diversas expresiones del turismo, contribuyendo a borrar la fragmentación categorial entre lo turístico y lo no turístico, lo cual significa y redimensiona que a través del turismo se puede analizar la realidad social, política y económica de una sociedad en un momento determinado. 